\title{
ANÁLISE TÉCNICO-ECONÔMICA DE DIFERENTES NÍVEIS DA TORTA DE MACAÚBA EM DIETAS PARA VACAS LEITEIRAS
}

\author{
TECHNICAL AND ECONOMIC ANALYSIS OF DIFFERENTS LEVELS \\ OF MACAUBA CAKE IN DIETS FOR DAIRY COWS
}

\author{
Azevedo, R.A. ; Bicalho, F.L. ${ }^{1}$; Araújo, L. ${ }^{1}$; Ribeiro Jr., C.S. ${ }^{1}$; Santos, A.C.R. ${ }^{1}$; Jayme, D.G. ${ }^{2}$ \\ e Geraseev, L.C. ${ }^{*}$
}

IInstituto de Ciências Agrárias. Universidade Federal de Minas Gerais. MG. Brasil. *Igeraseev@gmail.com ${ }^{2}$ Escola de Veterinária. Universidade Federal de Minas Gerais. MG. Brasil.

\author{
Palavras chave adicionais \\ Coprodutos. Custos com alimentação.
}

\section{RESUMO}

O preço do leite não acompanha a tendência de alta dos insumos e a redução dos custos de produção pode permitir a eficiência econômica da atividade leiteira. Dentro deste contexto, a utilização de coprodutos, pode ser alternativa para redução dos custos da alimentação e consequentemente os de produção. Objetivou-se analisar a inclusão de diferentes níveis da torta de macaúba, obtido na cadeia do biodiesel, na dieta total de vacas Holandesas, em lactação, em substituição ao farelo de milho. Foram utilizados oito animais com peso vivo médio de $480 \mathrm{~kg}$ e produção média de $25 \mathrm{~kg}$ de leite/dia, em dois quadrados latinos $4 \times 4$, sendo quatro níveis de inclusão da torta na matéria seca $(0,10,20$ e $30 \%)$ e quatro períodos de 21 dias, 14 de adaptação e sete dias de avaliação. Os animais foram alimentados ad libitum, com silagem de milho e concentrado, confinados em baias individuais. Avaliou-se o consumo de matéria seca total, produção de leite, custos diários e o retorno econômico das dietas. A inclusão do coproduto reduziu o consumo de matéria seca total e produção de leite, entretanto, a taxa de retorno marginal com $10 \%$ de inclusão do coproduto foi superior aos demais tratamentos, indicando que é uma alternativa técnica e economicamente viável em substituição ao farelo de milho na dieta de vacas leiteiras.

\section{SUMMARY}

The price of milk does not follow the upward

\author{
ADDITIONAL KEYWORDS \\ Coproducts. Feed costs.
}

trend of inputs so, reduction of production costs may enable economic efficiency of dairy farming. Within this context, the use of coproducts with highlights to those obtained in the biodiesel chain, may be an alternative for lower feed costs and consequently the production costs. The objective was to analyze the inclusion of different levels of the macauba cake in the total diet of lactating Holstein cows, to replace the corn meal. Eight animals with average weight of $480 \mathrm{~kg}$ and average production of $25 \mathrm{~kg}$ milk/day were used, in two $4 \times 4$ latin squares, with four levels of the cake $(0,10,20$ and $30 \%$ of dry matter) and four periods of 21 days, 14 to adapt and seven days for evaluation. The animals, kept in individual stalls, were fed ad libitum corn silage and concentrate. We evaluated the total dry matter intake, milk production, costs and economic returns of daily diets. The inclusion of coproduct in the diets reduced the dry matter intake and milk production, however, the marginal rate of return of $10 \%$ with the inclusion of coproduct was superior to the other treatments, indicating that the inclusion of 10 $\%$ macauba pie is a technically and economically feasible to replace corn bran diet in the diet of dairy cows.

\section{INTRODUÇÃO}

Ao considerar que as perspectivas futuras da valorização do preço do leite não acompanham a tendência de alta do preço 
dos insumos, a redução dos custos de produção pode ser uma alternativa para o produtor continuar na atividade de forma econômica, e a utilização de coprodutos pode reduzir os custos de alimentação, substituindo, em partes, os principais ingredientes utilizados tradicionalmente na dieta de animais em lactação (Souza et al., 2006), com destaque para aqueles obtidos na cadeia produtiva do biodiesel, como a torta da macaúba (TM) (Acrocomia aculeata). A TM é o coproduto da extração de óleo do fruto da macaúba e sua utilização vem sendo feita, sem avaliações de desempenho e de custo nas dietas de diferentes espécies de animais na região do Norte de Minas Gerais, local onde existe disponibilidade desse coproduto (Azevedo et al., 2012).

Objetivou-se com este trabalho realizar uma análise técnico-econômica do uso de diferentes níveis de inclusão da torta da macaúba em substituição ao farelo de milho na dieta de vacas da raça Holandesa em lactação.

\section{MATERIALE MÉTODOS}

O experimento foi conduzido no Instituto de Ciências Agrárias da Universidade Federal de Minas Gerais, Montes Claros, Minas Gerais, Brasil. Foram confinadas oito vacas da raça Holandesa, na segunda lactação, com peso vivo médio inicial de $480 \pm 26 \mathrm{~kg}$ e produção média $24 \pm 5 \mathrm{~kg}$ de leite/dia a partir dos 45 dias de lactação, distribuídas em dois quadrados latinos $4 \times 4$, quatro tratamentos e quatro períodos experimentais de 21 dias, com delineamento metodológico similar a Martins et al. (2011), sendo 14 dias de adaptação e 7 dias para avaliação do consumo de matéria seca total (CMST) e produção de leite (PL).

Os tratamentos foram quatro níveis $(0$, 10,20 e $30 \%$ ) de inclusão de TM na MS total das dietas, com relação entre concentrado e volumoso de $50 \%$ para cada. Os animais foram alimentados duas vezes ao dia, ad libitum, com dietas para atender as exigências de mantença e de produção de vacas com média de $500 \mathrm{~kg}$ de peso vivo, produzindo $25 \mathrm{~kg}$ de leite/dia, com um teor de gordura e proteína no leite de 3,4 e 4,0\%, respectivamente (NRC, 2001), sendo ordenhados diariamente com ordenhadeira mecânica, duas vezes ao dia, registrando-se a PL em balança digital. Análises químicobromatológica de MS das dietas (oferecido e sobras) de cada animal, por cada período, foram determinadas conforme Silva e Queiroz(2002).

Os custos de alimentação foram obtidos considerando o consumo dos ingredientes das dietas e os preços da silagem de milho ( $\mathrm{R} \$ 0,28)$, farelo de milho ( $\mathrm{R} \$ 0,41)$, farelo de soja $(\mathrm{R} \$ 0,66)$, uréia + AS $1 \%(\mathrm{R} \$ 1,25)$, calcário calcítico ( $\mathrm{R} \$ 0,17)$, fosfato bicálcico $(\mathrm{R} \$ 2,04)$ e premix mineral e vitamínico $(\mathrm{R} \$ 3,20)$, conforme levantamento do histórico de cotação do Centro de Estudos Avançados em Economia Aplicada da ESALQ/USP(CEPEA, 2010). A TM(R\$0,32) e o caroço de algodão $(\mathrm{R} \$ 0,34)$ foram cotados de acordo com os preços praticados na região de estudo, durante o período experimental. Outros custos não foram levados em conta, por não constituírem fatores de diferenciação.

Os custos foram avaliados segundo metodologia proposta por Rennó et al. (2008): custo total com o volumoso (CTV, multiplicação da quantidade de volumoso pelo seu valor de mercado), custo total de concentrado (CTC, multiplicação das quantidades de cada ingrediente que compunham o concentrado das dietas pelo seu respectivo valor de mercado), custo total da dieta (CTD, somatório do custo do concentrado e custo do volumoso) e custo total com alimentação (CTA, multiplicação do CMST pelo custo total da dieta).

Posteriormente foram avaliadas as receitas com a venda do leite por tratamento, utilizando-se o preço do leite referente ao valor médio pago no Estado de Minas Gerais, no mês de abril de 2010 ( $\mathrm{R} \$ 0,70)$, sendo 


\section{ANÁLISE TÉCNICO-ECONÔMICA DA TORTA DE MACAÚBA PARA VACAS LEITEIRAS}

avaliadas por meio das seguintes variáveis: renda bruta da produção de leite (RBPL) e receita menos os custos com alimentação (RMCA- diferença entre a renda bruta da produção de leite e o custo total com alimentação). Além das variáveis citadas, calculou-se a taxa de retorno marginal (TRM) conforme metodologia proposta por Evans (2005). As variáveis de CMST e PL foram analisadas por meio de análise de regressão em função dos níveis de torta de macaúba na dieta, utilizando-se o programa estatístico SAS (2002, versão 9.0).

\section{RESULTADOSEDISCUSSÃO}

A inclusão de torta de macaúba na dieta reduziu de forma quadrática $(\mathrm{p}<0,05)$ o consumo de MS, sendo o ponto de máxima do consumo ocorreu com $0,5 \%$ de inclusão do coproduto nas dietas, o que pode ser explicado pelo aumento nos teores de extrato etéreo e de fibra em detergente neutro das dietas com inclusão da TM. O comportamento quadrático do CMS refletiu diretamente na PL, a qual também apresentou comportamento quadrático (tabela I), sendo observado ponto de máxima com 1,3\% de inclusão da TM.

Na tabela I pode ser observado que o custo total do concentrado, da dieta e de alimentação reduziu com a inclusão da TM nas dietas, devido ao menor preço do coproduto quando comparado com o milho, principal ingrediente substituído nas dietas. O valor médio dos três tratamentos com a inclusão da TM para o custo total com concentrado foi de $\mathrm{R} \$ 0,48 / \mathrm{kg}$ de concentrado. Souza et al. (2006), ao avaliarem coprodutos em dietas de vacas em lactação, obtiveram custos próximos ao encontrado, com valores de $\mathrm{R} \$ 0,42$ por kg de concentrado composto por $15 \%$ de inclusão de casca de café.

O resultado observado para receita bruta de produção de leite por animal com a inclusão do coproduto reflete diretamente o resultado verificado para a PL, sendo que a maior receita foi verificada para os animais alimentados com $10 \%$ de inclusão de TM.

$\mathrm{O}$ aumento da receita bruta de produção de leite para o tratamento com $10 \%$ de TM refletiu na margem líquida (RMCA), sendo observado maior retorno para este tratamento em relação aos demais, demonstrando o potencial de utilização do coproduto. Segundo Davidson (1990) o uso de concentrado está diretamente correlacionado com a margem líquida, sendo esta crescente à medida que o preço do concentrado é menor

Tabela I. Consumo de matéria seca (CMST), produção diária de leite por animal (PL), custo por $\mathrm{kg}$ de volumoso (CTV), custo por $\mathrm{kg}$ de concentrado (CTC), custo por $\mathrm{kg}$ de dieta (CTD), custo total com alimentação por animal dia (CTA), receita bruta com a produção diária de leite por animal (RBPL), receita menos o custo com alimentação por animal/dia (RMCA) e taxa de retorno marginal (TRM) de diferentes níveis de inclusão de torta de macaúba na dieta total de vacas leiteiras. (Dry matter intake (CMST), daily milk production by animal (PL), cost per $\mathrm{kg}$ of forage (CTV), cost per kg of concentrate (CTC), cost per $\mathrm{kg}$ of diet (CTD), total cost of daily feeding animals (CTA), gross daily production of milk per animal (RBPL), revenue minus the cost of feed per animal/ day (RMCA) and rate of marginal return (TRM) of different inclusion levels of macauba cake in the total diet of dairy cows).

\begin{tabular}{lcccc}
\hline \multirow{2}{*}{ Variáveis } & \multicolumn{4}{c}{ Níveis de inclusão da TM } \\
& $0 \%$ & $10 \%$ & $20 \%$ & $30 \%$ \\
\hline CMST $^{1 a}$ & 18,36 & 18,41 & 16,69 & 15,19 \\
PL $^{1 b}$ & 23,22 & 23,92 & 19,95 & 18,44 \\
CTV $^{2}$ & 0,28 & 0,28 & 0,28 & 0,28 \\
CTC $^{2}$ & 0,50 & 0,49 & 0,48 & 0,47 \\
CTD $^{2}$ & 0,39 & 0,38 & 0,38 & 0,37 \\
CTA $^{3}$ & 7,17 & 7,08 & 6,34 & 5,69 \\
RBPL $^{3}$ & 16,25 & 16,74 & 13,97 & 12,91 \\
RMCA $^{3}$ & 9,09 & 9,66 & 7,63 & 7,22 \\
TRM (\%) $^{2}$ & $-663,33$ & 274,32 & 63,07 & 0 \\
\hline
\end{tabular}

${ }^{1} \mathrm{~kg} / \mathrm{animal} / \mathrm{dia} ;{ }^{2} \mathrm{~kg} \mathrm{MS}(\mathrm{R} \$) ;{ }^{3}$ animal/dia $(\mathrm{R} \$)$.

$\mathrm{a} Y=18,46-0,0042 x-0,0039 x^{2}\left(R^{2}=49 \%\right)$.

${ }^{\mathrm{b}} \mathrm{Y}=23,58-0,0146 \mathrm{x}-0,0056 \mathrm{x}^{2}\left(R^{2}=73 \%\right)$. 


\section{AZEVEDO, BICALHO, ARAÚJO, RIBEIRO JR., SANTOS, JAYME E GERASEEV}

e/ou a produção de leite for maior. No presente trabalho, embora tenha ocorrido redução no custo total do concentrado com a inclusão da TM, também ocorreu redução da PL nos tratamentos com 20 e $30 \%$ de TM, o que justifica menor margem líquida obtida nestes tratamentos.

A taxa de retorno marginal referente a inclusão da torta de macaúba foi calculada a partir do tratamento com $30 \%$ de inclusão de TM, por este ser o tratamento de menor custo. Essa taxa representa a diferença obtida com o incremento no retorno em porcentagem do custo total adicional (Evans, 2005) e permite concluir que a redução da inclusão do coproduto na dieta resultou em aumento na TRM até $10 \%$ de inclusão de TM, tratamento onde a mesma

\section{BIBLIOGRAFIA}

Azevedo, R.A.; Rufino, L.M.A.; Santos, A.C.R.; Silva, L.P.; Bonfá, H.C.; Duarte, E.R. e Geraseev, L.C. 2012. Desempenho de cordeiros alimentados com inclusão de torta de macaúba na dieta. Pesqui Agropecu Bras, 47: 1663-1668.

CEPEA. 2010. Centro de estudos avançados em economia aplicada da ESALQ/USP. <http:// www.cepea.esalq.usp.br/> (12/11/2010).

Davidson, T.M. 1990. The milk production potential of forage-concentrate systems in Queensland. High production per cow seminar. Queensland Department of Primary Industries. Sidney. pp. 1-13.

Evans, E.A. 2005. Análisis marginal: Un procedimiento económico para seleccionar tecnologías o prácticas alternativas. <http://edis.ifas.ufl. edu/pdffiles/FE/FE57300.pdf> (22/09/2012).

Martins, S.C.S.G.; Rocha Júnior, V.R.; Caldeira, L.A.; Pires, D.A.A.; Barros, I.C.; Sales, E.C.J.; Santos, C.C.R.; Aguiar, A.C.R. e Oliveira, C.R. 2011. Consumo, digestibilidade, produção de leite e análise econômica de dietas com diferen- apresentou resultado máximo $(274,32 \%)$. Ao comparar a TRM do tratamento controle com o tratamento com $10 \%$ de inclusão do coproduto, podemos observar que a TRM foi negativa indicando que a utilização do coproduto apresenta viabilidade econômica.

\section{CONCLUSÃO}

Apesar da redução dos parâmetros de consumo de matéria seca e de produção de leite, a taxa de retorno marginal do tratamento com $10 \%$ de inclusão de torta de macaúba foi superior ao tratamento controle, indicando que o coproduto é uma alternativa técnica e economicamente viável para substituição do milho na dieta de vacas leiteiras.

tes volumosos. Rev Bras Saúde Prod Anim, 12: 691-708.

NRC. 2001. Nutrient requirements of dairy cattle. National Research Council. $7^{\text {th }}$ ed. National Academy Press. Washington, DC. 381 pp.

Rennó, F.P.; Pereira, J.C.; Leite, C.A.M.; Rodrigues, M.T.; Campos, O.F.; Fonseca, D.M. e Rennó, N.N. 2008. Eficiência bioeconômica de estratégias de alimentação em sistemas de produção de leite: produção por animal e por área. Rev Bras Zootecn, 37: 743-753.

Silva, D.J. e Queiroz, A.C. 2002. Análise de alimentos (métodos químicos e biológicos). $3^{\underline{a}}$ ed. Imprensa Universitária. Viçosa.

SAS. 2002. Statistical analysis system. User guide: Stat. V. 9.0. SAS Institute Inc. Cary. NC.

Souza, A.L.; Garcia, R.; Valadares, R.F.D.; Pereira, M.L.A.; Cabral, L.S. e Valadares Filho, S.C.V. 2006. Casca de café em dietas para vacas em lactação: balanço de compostos nitrogenados e síntese de proteína microbiana. Rev Bras Zootecn, 35: 1860-1865. 